\title{
Gamma Knife patients' experience: lessons learned from a qualitative study
}

\author{
Wendy Clifford $\cdot$ Heather Sharpe $\cdot$ Kathleen Joy Khu $\cdot$ Michael Cusimano \\ Eva Knifed · Mark Bernstein
}

Received: 21 November 2008 / Accepted: 23 February 2009

(C) The Author(s) 2009. This article is published with open access at Springerlink.com

\begin{abstract}
Background Gamma Knife stereotactic radiosurgery (GKSRS) is an outpatient radiation therapy procedure in which a highly focused dose of radiation is delivered in a single fraction. It is used to treat a variety of well-demarcated intracranial lesions, including brain tumors. This study aims to explore patients' perspectives of the GKSRS process and the various stages involved. Methods Qualitative research methodology was used. Three semi-structured, open-ended interviews were conducted with 29 participants, who were ambulatory adult patients who underwent GKSRS between August 2007 and August 2008. Results Seven overarching themes emerged from the data: (1) patients were satisfied with the overall treatment experience; (2) the majority of patients had a good knowledge of GKSRS; (3) the quality and amount of patient education were adequate; (4) process expectations were largely met; (5) most patients prioritized outcome over process; (6) most patients had a realistic expectation of outcomes; and (7) pain and anxiety were important issues. Conclusions The study results reflected positively on the GKSRS process, but there were some areas identified that require improvement, specifically the referralconsultation process and the management of procedural
\end{abstract}

Electronic supplementary material The online version of this article (doi:10.1007/s11060-009-9830-7) contains supplementary material, which is available to authorized users.

W. Clifford - H. Sharpe - K. J. Khu - M. Cusimano - E. Knifed ·

M. Bernstein ( $\square)$

Division of Neurosurgery, Toronto Western Hospital,

University Health Network, University of Toronto,

399 Bathurst Street, 4 West Wing, Toronto,

ON M5T 2S8, Canada

e-mail: mark.bernstein@uhn.on.ca pain. These insights on the patients' perspectives can lead to better delivery of care and ultimately, improved health outcomes.

Keywords Gamma Knife - Qualitative research · Radiosurgery

\section{Introduction}

Stereotactic radiosurgery (SRS) has become an indispensable part of the neuro-oncologist's armamentarium, mainly for treatment of metastatic tumors and vestibular schwannomas. Gamma Knife radiosurgery (GKSRS) is a form of radiosurgery that allows the treatment of discrete lesions with high conformality using three-dimensional stereotactic imaging and the delivery of multiple radiation beams through collimators with the target lesion as the focal point $[1,2]$. It is used to treat a variety of conditions-mainly benign and malignant tumors, vascular lesions, and functional disorders. As a result, there is diversity in patient needs, expectations, and experiences.

Even if the treatment is instituted within a single day, there are multiple stages involved in the GKSRS treatment process. These include the referral process, multi-disciplinary conference for review of cases, consultation, education, treatment, and follow-up.

Thousands of studies have been published regarding clinical results and outcomes of patients who have undergone GKSRS, but very few have dealt with patients' perspectives [3-6]. This qualitative study was undertaken to explore in-depth patients' experiences and learn their perspectives at various stages of the GKSRS process, from the initial referral to treatment to follow-up. 


\section{Methodology}

Study design

This study was a qualitative research study using interviews with patients who received GKSRS treatment for a variety of intracranial conditions.

\section{Setting and participants}

Participants were patients who underwent GKSRS at the University Health Network Gamma Knife. They were ambulatory adult patients age 18 or older and were proficient in English. The study objective was discussed with each patient, and consent was obtained prior to the commencement of the first interview. Pediatric patients and those who required a translator to communicate were excluded. Printed educational materials were provided to each patient after the first interview (see Appendices provided in the Supplementary material).

\section{Sample size}

Approximately 25-30 participants were sought for the study. This range was selected because most qualitative studies reach "saturation" at these numbers. Saturation is the point beyond which no new concepts arise from subsequent patient interviews [7]. Twenty-five to 30 is the number of subjects used in most surgical qualitative studies [7].

\section{Data collection}

Three semi-structured, open-ended interviews were scheduled for each patient. The interviews took place at the following stages: (1) the first one was after the consultation at the Gamma Knife clinic but before the education session; (2) the second one was on the day before treatment; and (3) the third one was 3 months following GKSRS treatment. The first and third interviews were conducted face-to-face with the patient, whereas the second interview was carried out over the telephone. The interviews were based on a guide, but themes were freely explored as they arose. All interviews were audio taped and transcribed. Demographic parameters were collected for each patient.

Data analysis

The responses to all questions from all participants were first collected in tabular form and then interview data were examined through modified thematic analysis, which includes open and axial coding [7]. Open coding involves breaking down information into common groupings based on shared ideas, while axial coding involves organizing information according to overarching themes. The transcriptions were analyzed by five investigators, who all contributed to developing the coding framework.

Research ethics

All data gathered for this study were kept confidential. The audiotapes and anonymized transcriptions were kept in a secure location. Participation in the study was completely voluntary, and informed consent was obtained from all participants. The study was approved by the Research Ethics Board of the University Health Network.

\section{Results}

Patient information

Twenty-nine patients were recruited using convenience sampling over a 1 year period between August 2007 and August 2008. During this period, 242 patients were treated with GKSRS: 141 on the 4C Gamma Knife unit and 101 on the Perfexion unit. Thus, the study sample represented $12 \%$ (29/242) of all treated patients. Of the 29 patients, 28 first interviews, 21 second interviews, and 22 third interviews were obtained. The patients' demographic data are shown in Table 1.

\section{Thematic analysis}

Seven overarching themes emerged from the analysis of the interviews. These themes are briefly described and illustrated using verbatim quotes from the patient interviews (as is the norm when reporting qualitative research).

Table 1 Demographic data of the study participants

\begin{tabular}{ll}
\hline Age (years) & \\
Range & $29-83$ \\
Mean & 63.1 \\
Sex & \\
$\quad$ Male & $41 \%$ \\
Female & $59 \%$ \\
Education & \\
Post-secondary & $73 \%$ \\
Secondary & $13 \%$ \\
Public school & $13 \%$ \\
Diagnosis & \\
Non-malignant tumour & $38 \%$ \\
Malignant tumour & $28 \%$ \\
Trigeminal neuralgia & $21 \%$ \\
Tremor & $10 \%$ \\
AVM & $3 \%$ \\
\hline
\end{tabular}


1. Patients were satisfied with the overall treatment experience

All the patients who received treatment expressed satisfaction with the overall experience, in spite of the fact that $59 \%$ of patients complained of transient worsening of symptoms or mild post-treatment complications. The majority of the patients stated that they were glad they went through with the treatment, and that they would recommend the same procedure to their family member or friend should he/she need it.

It was just like going to a spa but I had holes in my head...

I think that was the easiest treatment I ever had...

...very positive experience... well worth the discomfort...

It's not as bad as it looks.

Factors that helped patients get through the treatment include trust in the physician and other members of the Gamma Knife team, sufficient knowledge about the procedure and knowing what to expect, self-fortitude, and family. Patients also remarked that the staff were friendly, caring, and competent, which helped to make them feel comfortable and put their mind at ease.

Some patients complained about the time lag in between procedures such as frame fitting and the actual treatment.

The whole day was waiting around...

However, most patients were not bothered by the periods of waiting during the day because they were advised about it beforehand, and thus, expected it. They also understood the reason for the time lag, which was used mainly for treatment planning.

No [there wasn't too much waiting around], we were warned ahead of time that there would be a wait...

\section{Patients had a good knowledge of GKSRS}

The majority of patients knew their treatment options and understood the reason why they were referred for GKSRS. Most had a good concept of how GKSRS works and what it can achieve for them. The comments below illustrate the participants' definition of GKSRS. Some of them were concrete whereas others were more imaginative.

It is... 201 points of radiation focused on a single area.

...where you've had a magnifying glass and burned a leaf... It doesn't damage anything going through but at the end of that point, is where it's doing what it's supposed to do. ...focused radiation on tumors... kind of like Star

Wars. They blast them out of the sky!

\section{The quality and amount of patient education was adequate}

Most of the patients felt that the amount of information provided was quite thorough. This included printed pamphlets, and information provided by the doctors in the Gamma Knife team who saw the patient on initial and subsequent consults, as well as the radiation therapists and nurses who conducted the education session.

I feel very comfortable. I didn't expect them to be that informative, actually, so it's good for me, I feel more comfortable now.

Although most of the participants already had a fair idea of GKSRS even prior to the first consult, an overwhelming majority felt that their understanding of GKSRS was enhanced after the education session. The educational video was deemed extremely helpful, especially for the visual learners. Printed material in the form of patient brochures and pamphlets were also appreciated. Some patients suggested direct visualization or photographs of the frame and the machine, as well as testimonials of patients who had already undergone GKSRS, as adjuncts to improve their understanding.

\section{Process expectations were largely met}

The majority of patients chose GKSRS because it was suggested by their physician. There were also other factors such as reluctance to undergo an operation or failure of medical treatment. Only four patients (14\%) discovered GKSRS during the course of their own research and persuaded their physician to make the referral for them.

A total of $71 \%$ of patients felt that the referral-consultation process occurred in a timely manner. The definition of "timely" varied from patient to patient, but people were quite reasonable ("I'm not the only one... with a brain tumor"). The $29 \%$ who felt that there was a delay experienced increased anxiety during the waiting period. The latter group suggested an earlier appointment, improved communication between doctors' offices, and encouraging self-referral by increased advertisement of GKSRS, to decrease the waiting time before consultation at the Gamma Knife clinic.

Most patients expected information, both factual and logistic, during their consultation at the Gamma Knife clinic. Patients were satisfied with the consult, in that they received the information they needed and had their questions answered. Patients were pleased with the team approach because they felt that they were getting better care. 
... and the other doctors, we know they're all part of the team. I think probably it helped to make us more confident and more comfortable... it appears that extreme care and consideration is [sic] being given for this procedure.

Some patients remarked that they would have preferred having the follow-up visit earlier than 3 months, and a small number of patients complained that they did not receive any post-treatment telephone call as they were led to believe would happen.

\section{Patients prioritized outcome over process}

It was quite clear that most patients had a singular interest in outcome that took priority over the process issues. They did not lose sight of the big picture, and felt that the discomfort they went through was negligible if it meant an improvement in their primary disease.

You have to keep the outcome... the goal... in mind.

You know, many years versus one day...

\section{Patients had a realistic expectation of outcome}

The majority of patients had realistic expectations of what GKSRS can do for them, although a small minority had misconceptions about the treatment outcome ("...lifetime solution to a problem"). Most patients with non-life threatening conditions understood, however, that GKSRS was expected mainly to improve one's quality of life instead of length of life. Most patients also understood that the treatment effect may not manifest immediately, and may, in fact, take months to years.

takes a long time for the effects to show... six months to one year...

In spite of understanding this concept, some patients still felt frustration at the helplessness and uncertainty caused by the long waiting time.

\section{It's just a process that takes so long to find out that it} actually worked...

Because of this, patients who felt no improvement in their symptoms were cautious and sensible enough to reserve judgment and say that it was still too early to tell if their desired outcomes have been met.

\section{Pain and anxiety were important issues}

A total of $63 \%$ of patients complained of pain when the frame was placed, and an equal number experienced pain when the frame was removed. A total of $59 \%$ complained of headache or scalp pain which persisted for days to weeks after the procedure.

I felt like I had a severe sun burn all over my scalp...

In spite of the consultation and the education session, $36 \%$ of patients felt that they were not prepared for the pain from the frame placement. Most of them remarked that there was nothing that could have prepared them anyway.

The procedure was very traumatic... didn't realize the pins would hurt that much...

Many patients expressed anxiety during the pre-treatment interview, but surprisingly, the anxiety began to fade once the frame was placed and the treatment commenced. The only ones who remained anxious were the handful of claustrophobic patients, but their fears were addressed by giving them sedatives during the procedure.

\section{Discussion}

Gamma Knife Radiosurgery is so much less invasive than the surgical alternative, that the risks and discomforts may be minimized by health care providers and patients alike. Most physicians GKSRS consider it a much more minor procedure than surgery-this is especially true for neurosurgeons. To understand any potential negative aspects of GKSRS and to truly explore the patients' full experience, we must ask our patients for narratives descriptions of their experience. This is the core of qualitative inquiry. This also represents a form of quality assurance or audit that is integral to improvement of any health care program.

The current study was unique in that we focused on GKSRS patients' experience using qualitative in-depth openended interviews. In fact, each patient participated in three interviews: following the consultation appointment, the day before the scheduled GKSRS, and 3 months post GKSRS.

Despite the somewhat predictable themes, the findings showcase patient sentiments of an entire medical process. The sample size also provided us with an assortment of diagnoses, both life threatening and non-life threatening, ages, patient educational levels, and ethnic backgrounds. Not surprisingly, the predominant theme that emerged was satisfactory with the entire process. Patients expressed a high level of satisfaction with the care they received at the various stages of the process.

The referral-consultation process was felt to occur in a timely manner for most participants. For patients with benign and stable conditions, urgency of the appointment was not an issue as long as it occurred within a reasonable period. However, for patients with malignant conditions or those associated with progressive neurological deficit or 
severe pain, the time line was not considered fast enough. Gamma Knife patients are prioritized according to the level of urgency, and the patients who benefited from this practice were appreciative of the fact that they were seen in clinic within a week or two of the referral.

In terms of pre-consultation knowledge of GKSRS, two types of patients were identified. The first type were those who came to the consultation very well-informed, armed with knowledge from the internet, support groups, or other sources. The second group of patients was less selfempowered and preferred to leave the decision in the hands of their physician. They had trust in their doctor and the health system, and did not feel the need to seek information on their own. These were often elderly patients accompanied by a family member who was the information seeker.

Education was felt to be adequate; patients felt satisfied with the information obtained from the physicians and allied health care workers and from the education session. Written and visual (video) materials were appreciated by most patients. However, most of the information imparted dealt with process issues instead of outcome issues, which was what some of the patients wanted to know. There were also some sentiments that widespread knowledge of GKSRS required improvement among community health practitioners. This likely reflects the relative youth of the Ontario Gamma Knife program which only started in September 2005, although linear accelerator radiosurgery has been available in Ontario for over 20 years. This study was therefore conducted during the third year of operation of the Toronto Gamma Knife.

Treatment day expectations varied amongst patients, with several participants expressing anxiety about the procedure. Pre-treatment anxiety can be categorized into "fears of the unknown" and "fears of illness" [8]. Fears of the unknown include patient dependence on the doctor during the treatment procedure, a lack of knowledge with respect to what occurs during treatment, and uncertainty about outcome. On the other hand, fears of illness include fears of post-treatment complications and pain, mental and physical harm caused by the procedure, and disease-specific issues [8]. In the case of GKSRS, patients fear both the condition being treated, and the prospect of a complex high-tech treatment [3]. These issues were paramount in their thoughts during treatment day, and feelings of anxiety were common in other GKSRS patients as well $[3,6]$.

For the patients, a distinctive and memorable part of the whole process is the placement of the stereotactic frame on the head at the beginning of the day. Since it was the only invasive part of the procedure, pin-site pain figured prominently in the patients' narratives. Many patients also complained of pain after frame removal, which continued on as headaches that lasted for hours to days. This is consistent with other findings in the literature regarding acute symptoms after GKSRS. St. George et al. [5] reported that $50 \%$ of patients complained of post-treatment headache, whereas $33.3 \%$ complained of pin site pain or numbness. Other authors [9-13] also reported findings of headaches although their papers focused on more severe symptoms such as seizures and neurologic deficits. Rozen and Swanson [14] proposed a distinct headache entity called "post Gamma Knife headache" since they observed a de novo headache syndrome in a number of patients who underwent GKSRS for cerebral arteriovenous malformation (AVM).

While the results of this study reflect positively on the GKSRS process, patients have provided us with information on areas requiring improvement. The referralconsultation process caused anxiety in some patients, thus, efforts need to be made to relieve or minimize these feelings. This can be done by streamlining the process and increasing communication to the patient. Increased education of community physicians to facilitate widespread knowledge of GKSRS must be within the mandate of all Gamma Knife units as they begin their operations. Pain issues need to be addressed and improved, for both patient satisfaction and patient comfort.

The findings from this study gave us an expanded view of the process from the patient's perspective, information not attainable by quantitative research methodology. This information can lead to enhanced empathy between patient and health care provider and ultimately better health outcomes. Many clinicians with extensive experience with GKSRS may have difficulty resonating with the few negative findings in this study (especially frame discomfort), but we respectfully submit that if patients are specifically asked about their experience, we clinicians will often hear some unexpected results which can help us do our jobs better.

Acknowledgments We gratefully acknowledge the generosity and kindness of the patients and all the staff in the Gamma Knife unit at UHN. We also acknowledge two grants: (1) Canadian Institutes of Health Research, MOP 77670, Therapeutic Hopes and Ethical Concerns: Clinical Research in the Neurosciences; and (2) Canadian Institutes of Health Research, NNF 80045, States of Mind: Emerging Issues in Neuroethics for help supporting this work.

Open Access This article is distributed under the terms of the Creative Commons Attribution Noncommercial License which permits any noncommercial use, distribution, and reproduction in any medium, provided the original author(s) and source are credited.

\section{References}

1. Kondziolka D, Lunsford D, Flickinger JC (2008) The application of stereotactic radiosurgery to disorders of the brain. Neurosurgery 62(suppl 2):707-719. doi:10.1227/01.neu.0000316275. 12962.0e

2. Barnett GH, Linskey ME, Adler JR, Cozzens JW, Friedman WA, Heilbrun MP, Lunsford LD, Schulder M, Sloan AE, The American 
Association of Neurological Surgeons/Congress of Neurological Surgeons Washington Committee Stereotactic Radiosurgery Task Force (2007) Stereotactic radiosurgery—an organized neurosurgery-sanctioned definition. J Neurosurg 106:1-5. doi:10.3171/jns. 2007.106.1.1

3. Menkes DB, Davison MP, Costello SA, Jaye C (2005) Stereotactic radiosurgery: the patient's experience. Soc Sci Med 60: 2561-2573. doi:10.1016/j.socscimed.2004.11.021

4. Nichols DO (2006) Patient perception of disability following acoustic (vestibular) schwannoma removal via gamma knife and microsurgical techniques. Available at: http://hdl.handle.net/ 1838/523. Accessed Nov 2008

5. St George EJ, Kudhail J, Perks J, Plowman PN (2002) Acute symptoms after gamma knife radiosurgery. J Neurosurg 97(5): 631-634

6. Ward-Smith P (1997) Stereotactic radiosurgery for malignant brain tumors: the patient's perspective. J Neurosci Nurs 29:117-122

7. Bernstein M, Potvin D, Martin DK (2004) A qualitative study of attitudes towards error in patients facing brain tumor surgery. Can J Neurol Sci 31:208-212

8. Walker JA (2002) Emotional and psychological preoperative preparation in adults. Br J Nurs 11:567-575

9. Chin LS, Lazio BE, Biggins T, Amin P (2000) Acute complications following gamma knife radiosurgery are rare. Surg Neurol 53:498-502. doi:10.1016/S0090-3019(00)00219-6
10. Singh VP, Kansal S, Vaishya S, Julka PK, Mehta VS (2000) Early complications following gamma knife radiosurgery for intracranial meningiomas. J Neurosurg 93(suppl 3):57-61

11. Sutcliffe JC, Forster DMC, Walton L, Dias PS, Kemeny AA (1992) Untoward clinical effects after stereotactic radiosurgery for intracranial arteriovenous malformations. Br J Neurosurg 6:177-185. doi:10.3109/02688699209002925

12. Werner-Wasik M, Rudoler S, Preston PE, Hauck WW, Downes BM, Leeper D, Andrews D, Corn BW, Curran WJ (1999) Immediate side effects of stereotactic radiotherapy and radiosurgery. Int J Radiat Oncol Biol Phys 43:299-304. doi:10.1016/ S0360-3016(98)00410-6

13. Vachrajani S, Fawaz C, Mathieu D, Menard C, Cusimano MD, Gentili F, Hodaie M, Kenny B, Kulkarni AV, Laperriere N, Schwartz M, Tsao M, Bernstein M (2008) Complications of Gamma Knife radiosurgery: an early report from two Canadian centers. J Neurosurg 109:2-7

14. Rozen TD, Swanson JW (1997) Post gamma knife headache: a new headache syndrome? Headache 37:180-183. doi:10.1046/ j.1526-4610.1997.3703180.x 